\title{
La imagen pública: estudio de caso en Camagüey (Cuba)
}

\author{
The public image: a case study \\ in Camagüey (Cuba)
}

\author{
MSc. Lesvia Amelia Prieto Valdivieso (Cuba) \\ lesvia.prieto@reduc.edu.cu \\ Universidad de Camagüey
}

\section{MSc. Karla Gómez González (Cuba) \\ Universidad de Camagüey}

\author{
Lic. Lirisdey Pimentel Dorta (Cuba) \\ Universidad de Camagüey
}

\section{Lic. Marcelo Chacón Reyes (Cuba)}

marcelo.chacon@reduc.edu.cu

Universidad de Camagüey

\section{Resumen}

Esta investigación aplica las subcategorías percepción, opiniones, actitudes y mediaciones sociales para medir la imagen pública y describe el caso particular de los resultados de la imagen pública que tienen los habitantes de la circunscripción 144 del Consejo Popular La Belén acerca de la recreación en su comunidad, y del Círculo Social Obrero "Pedro Soto Alba".

Se utiliza una combinación metodológica mixta (cualitativa y cuantitativa), así como técnicas de investigación /cuestionarios,

\section{Abstract}

This research applies the sub-categories of perception, opinions, attitudes and social mediations for measuring the public image, describe the particular case of the public image that the inhabitants of the Popular Council number 144 have about the recreation in their community and the Workers's Social Club "Pedro Soto Alba". It uses a combination of qualitative and quantitative methodologies, and techniques like questionnaires, interviews, observation, and documental analysis.
FECHA DE RECEPCIÓN:

FECHA DE REVISIÓN:

APROBACIÓN:
1 de abril de 2013

2 de julio de 2013

17 de septiembre de 2013
Para Citar este artículo / to Cite this ARticle Chacón, M., Gómez, K., Pimentel, L. \& Prieto, L. (2013). La imagen pública: estudio de caso en Camagüey (Cuba). Poliantea, (IX), 17, pp. 109-128. 
entrevistas, observación y análisis de documentos).

Los resultados muestran que los

habitantes no tienen una imagen pública acerca de la recreación y ven al Círculo Social Obrero "Pedro Soto Alba" como un espacio de diversión.

Palabras clave: imagen pública, percepción, actitudes, mediaciones sociales.
Results show that the inhabitants have no public image about the recreation in their community, and they see the Workers's Social Club "Pedro Soto Alba" as a space for recreation.

Key Words: public image, perception, opinions, attitudes, social mediations. 


\section{La imagen pública: estudio de caso en Camagüey (Cuba)}

\section{The public image: a case study in Camagüey (Cuba)}

MSc. Lesvia Amelia Prieto Valdivieso (Cuba)

lesvia.prieto@reduc.edu.cu

Universidad de Camagüey

MSc. Karla Gómez González (Cuba)

Universidad de Camagüey
Lic. Lirisdey Pimentel Dorta (Cuba)

Universidad de Camagüey

Lic. Marcelo Chacón Reyes (Cuba)

marcelo.chacon@reduc.edu.cu

Universidad de Camagüey

\section{Introducción}

La imagen ha sido objeto de investigación desde algunas disciplinas, entre ellas la sociología, la psicología y la comunicación social, las cuales han contribuido a conceptualizar la imagen desde diferentes aristas y clasificarla.

Entre los numerosos estudios sobre imagen con relación a la comunicación social en Cuba, son disímiles los temas que se han desarrollado; por ejemplo, sobre imagen corporativa podemos encontrar investigaciones como la "Auditoría de imagen de Transtur Rent a Car", de Abel Montero y Arianna Oviedo Bravo, en 2004, o el "Diagnóstico de imagen interna de la casa matriz del grupo empresarial extrahotelero Palmares", de Eyilen González Sera, de 2008. En Cuba también se han desarrollado estudios sobre la imagen de fenómenos y lugares, entre los que se incluyen "La tierra prometida... de cómo algunos jóvenes habaneros imaginan Cuba: el deseo o la certeza de país", de Rosa Muñoz (2003) y "Todos los caminos conducen al centro”, de Aylín Martínez Venegas y Dalila González García (2008).

Para este estudio se utilizó como antecedente la propuesta realizada por Alain de la Noval Bautista y Aliuska Milán Mariño en su tesis “Aproximación 
al diagnóstico de la identidad y la imagen del Programa de Trabajadores Sociales de Arroyo Naranjo", utilizado también por Magalis Díaz Alemán en su tesis de licenciatura "Mil palabras, una imagen, un acercamiento a la imagen pública del taller de transformación integral de barrio, de la comunidad Micro X, del Consejo Popular Alamar del Este".

En Camagüey (Cuba) son pocos los reportes de investigaciones relacionados con la imagen, y estos han estado basados principalmente en investigaciones acerca de la imagen corporativa, como es el caso de la "Estrategia de comunicación para fortalecer la autoimagen corporativa de la compañía Danza Contemporánea de Cuba", de Ernesto Suárez, y otra que le toca muy de cerca, relacionada con la identidad: "Estrategia de comunicación para fortalecer la identidad corporativa del Ballet de Camagüey", de Jany Montes de Oca, ambas en 2010. Hasta el momento, en esta provincia no se han realizado estudios sobre la imagen pública donde se combinen las disciplinas de la comunicación social, la psicología y la sociología.

La presente investigación se realiza en el Consejo Popular La Belén, del municipio de Camagüey, por ser un entorno priorizado para su transformación social debido a sus múltiples problemáticas, lo que obedece a un proyecto del Programa Territorial de Ciencias Sociales y Humanísticas que lleva a cabo el Centro de Estudios para el Trabajo Comunitario, de la Universidad de Camagüey. Una de las temáticas más importantes a resolver por este consejo popular lo constituye la recreación, uno de los satisfactores importantes dentro de la necesidad de ocio de las personas.

Para el ser humano es necesario el mejoramiento de su calidad de vida, expresión que se utiliza en una generalidad de contextos, tales como sociología, ciencia política, estudios médicos, estudios del desarrollo y otros.

La Organización Mundial de la Salud (OMS) la define como el estado de bienestar, felicidad y satisfacción de un individuo, que le otorga cierta capacidad de actuación, funcionamiento o sensación positiva de su vida. Su realización es muy subjetiva; se ve directamente influida por la personalidad y el entorno en el que vive y se desarrolla el individuo.

Junto con el concepto calidad de vida se estudia la recreación; cuando esta es adecuada, puede conseguir 
que las personas se sientan a gusto con sus vidas, aun con los problemas que se presentan a diario.

En las comunidades, las relaciones entre sus pobladores establecen las condiciones idóneas para el desarrollo de una recreación sana, que propicie bienestar, salud, y eleve el nivel cultural de sus miembros, siendo ellos los protagonistas mediante la participación comunitaria.

Para que la recreación sea efectiva los habitantes de la comunidad deben aportar las ideas, asílos líderes y las instituciones correspondientes lograrán realizar ofertas que cumplan con el verdadero propósito de elevar la calidad de vida de las personas, según los gustos y preferencias de los diversos grupos sociales.

En las comunidades también existen instituciones cuyo objeto social fundamental es proporcionar recreación, por lo que deben estar en constante interacción con los miembros de la comunidad y sus dirigentes, buscando brindar un mejor servicio y la finalidad de que las personas puedan usar su tiempo libre con recreación eficaz, que brinde diversión y a la vez contribuya a su mejoramiento humano. Sin duda la comunicación ha adquirido importancia en la modernidad y desempeña hoy un papel relevante en la sociedad.

El objetivo de esta investigación, por tanto, es el de describir la imagen pública que tienen los habitantes de la circunscripción 144 del Consejo Popular La Belén y del Círculo Social Obrero "Pedro Soto Alba", acerca de la recreación en sus comunidades.

\section{Marco teórico}

El interés por el concepto de imagen, tanto teórico como práctico, tiene su origen, principalmente, en el campo del márquetin. Esto es cierto tanto para la literatura académica como para los logros y publicaciones de los profesionales de la imagen (por ejemplo, Ogilvy en los años cincuenta). En los últimos cinco o diez años las ideas básicas que sobre la imagen se crearon, en un principio, en el campo del márquetin a nivel de producto, van extendiéndose a una escala organizativa (imagen corporativa) (Trelles, Meriño y Espinoza, 2005, p. 10).

Justo Villafañe (1985), catedrático de la Universidad Complutense de Madrid, consultor de reconocido prestigio en temas de gestión de comunicación e imagen, expresa: 
La imagen como representación es la conceptualización más cotidiana que poseemos y, quizás por ello, se reduce este fenómeno a unas cuantas manifestaciones. Sin embargo, comprende otros ámbitos que van más allá de los productos de la comunicación visual; implica también otros procesos como el pensamiento, la percepción, la memoria, en suma, la conducta (p. 29).

Los estudios acerca de la imagen son bastante recientes y han abundado principalmente en el término "imagen corporativa", aunque todavía no se tiene un concepto claro y coherente sobre su significado.

Dowling, en su artículo "Managing Your Corporate Image" (1986), siguiendo a Aaker y Myers (1982), sostiene:

Una imagen es el conjunto de significados por los que un objeto se conoce y a través del cual la gente lo describe, recuerda y relaciona. Es decir, es el resultado neto de la interacción de las creencias, ideas, sentimientos e impresiones que una persona tiene de un objeto (Trelles, Meriño y Espinoza, 2005, p. 5).

Usualmente la creación de una imagen es un proceso regulado, dirigido y puesto en marcha por un emisor que es, en última instancia, una institución.
Justo Villafañe considera que la imagen institucional está conformada por tres dimensiones: la autoimagen, la imagen intencional y la imagen pública, esta última la de más difícil conocimiento, pues es la dimensión que representa la percepción que el entorno tiene de una organización (Trelles, Meriño y Espinoza, 2005, p. 5).

Para el estudio de la imagen pública Magalis Díaz Alemán en su tesis propone utilizar dimensiones no provenientes de la comunicación social, debido a la complejidad de su medición.

Siguiendo esta perspectiva, los autores proponen la utilización de las subcategorías percepción, opinión, actitud y mediaciones sociales, aplicadas a la medición de la imagen pública de la recreación.

La imagen pública se concibe como la percepción que el entorno tiene de una organización; la percepción dominante que una colectividad establece respecto de un actor, institución o referente cultural, con base en las impresiones y la información pública que recibe (Trelles, Meriño y Espinoza, 2005, p. 5). Las subcategorías de análisis, percepción, opiniones, actitudes y mediaciones sociales que seguiremos en 
adelante serán vistas de acuerdo con los siguientes conceptos:

- Percepción: como la instancia mediadora entre el objeto exterior y el concepto que de él se hacen las personas -el reconocimiento del medio que las circunda- es la imagen mental formada con ayuda de la experiencia y las necesidades, es resultado de un proceso de selección, interpretación y corrección de sensaciones; un proceso cíclico, de carácter activo, constructivo, relacionado con procesos cognitivos superiores y que transcurre en el tiempo. Sin embargo, percibir no es recibir información visual pasivamente, pues implica buscar, seleccionar, organizar, relacionar, establecer conexiones, recordar, identificar, jerarquizar, evaluar, aprender e interpretar (Manzano, 2006).

- Opiniones: son consideradas una de las dimensiones válidas para puntualizar la definición de la imagen pública y tienen un carácter variable, pueden estar mediadas por las motivaciones y posiciones tomadas ante una situación o fenómeno; constituyen la toma de posición o partido frente a una cuestión en particular, teniendo en cuenta la relevancia que tengan para los individuos en general (Casales, 2009).

- Actitud: es la predisposición adquirida y relativamente duradera, a evaluar de determinado modo a una persona, suceso o situación y actuar en consonancia con dicha evaluación; es una orientación social, una inclinación subyacente a responder a algo de manera favorable o desfavorable; es un estado de ánimo. Se cataloga como cognitiva, afectiva y conductual (Casales, 2009).

- Mediaciones sociales: constituyen el conjunto de influencias cognitivas y socioculturales que estructuran el proceso de aprendizaje de los sujetos y sus resultados, provenientes tanto de la mente del sujeto como de su contexto sociocultural. Estas pueden ser individuales, textuales, contextuales o situacionales. Las mediaciones individuales son aquellas que tienen que ver con la historia personal y la vida diaria del sujeto, factores sociopsicológicos que se 
derivan de procesos cognitivos y afectivos, rasgos personológicos que influyen fundamentalmente en la construcción de significados, teniendo en cuenta la posición social, definida por la integración del sujeto en la estructura socioclasista, por su nivel de calificación, ocupación e ingresos económicos, todo lo cual determina su acceso a los recursos necesarios para el consumo cultural (Portal y Recio, 2003).

\section{Metodología}

La actual investigación es comunicológica, un estudio descriptivo transversal de tipo no experimental, que parte de una perspectiva cuantitativa-cualitativa. Las técnicas de la perspectiva cuantitativa utilizadas en la investigación fueron el cuestionario y el análisis de documentos. De la perspectiva cualitativa se aplica la observación participante y los informantes clave, a los cuales se le aplicó entrevistas en profundidad.

La circunscripción 144 del Consejo Popular La Belén, donde se encuentra el Círculo Social Obrero "Pedro Soto Alba", presenta un universo de 2631 habitantes. Se realiza la selección de las muestras tomando en cuenta los métodos de estudio. Para las entrevistas se seleccionan informantes clave, en este caso el administrador del Círculo Social Obrero, el delegado de la circunscripción y el trabajador social de la comunidad, de modo que $n=3$. Para las encuestas se tomó una muestra de $\mathrm{n}=78$ personas utilizando un muestreo intencional por cuotas para tener una representación de los dos sexos y de todas las edades, como se ilustra en la tabla 1 del anexo 1 .

\section{Resultados}

A continuación se presentan los resultados de la investigación por subcategorías de análisis.

\section{Percepción}

La recreación es percibida de diferentes maneras: mientras que $47.4 \%$ a quienes se les pidió que escribieran todas las palabras que se les vinieran a la mente cuando se les habló de recreación en su circunscripción, la relacionaron con jugar, planes de la calle, bailar, divertirse o intervenir en juegos participativos; $39.9 \%$ escribió que con nada la asociaban.

En entrevista al delegado de la circunscripción se conoció que se programan muchas actividades culturales para la comunidad, pero 
en muchas ocasiones faltan los recursos materiales. En las encuestas también se pudo conocer que la percepción que tienen los pobladores acerca de la recreación en su comunidad se basa principalmente en las acciones que se realizan en fechas conmemorativas como el 28 de septiembre, Día de los Comité de Defensa de la Revolución (CDR), el Día de la Mujer y otros, en las que se hacen intercambios de regalos y se llevan platos de dulces, entre otros.

En general el Círculo Social es visto como un lugar recreativo donde se puede "descargar" con los amigos, realizar actividades como celebraciones de cumpleaños, tomar cerveza, oír música, hacer relaciones sociales, todo al alcance de las posibilidades económicas de los habitantes de la comunidad, así lo jusga 59 \% de la población encuestada. Sin embargo, $24.4 \%$ lo percibe como un lugar sin condiciones para realizar actividades, pues el local se encuentra muy deteriorado y por tanto no sienten motivación para asistir; $14.1 \%$ lo considera "el lugar de los borrachos" porque aún queda un remanente de su mala reputación en tiempos pasados debido a peleas ocurridas entre sus asistentes, aunque en estos momentos dicha situación se ha erradicado y los entrevistados lo aprecian como un lugar tranquilo en el que se puede despejar la mente de los problemas cotidianos.

En entrevista realizada al administrador del lugar, este comenta:

La recreación es prácticamente nula, lo que se hace es venir a tomar cerveza y la comunidad necesita más que eso, como actividades para los niños y juegos de participación, entre otros; solo necesitamos un poco más de recursos, y que la comunidad utilice las áreas del lugar para implementar los planes que tienen.

En cuanto a las significaciones del lugar para las personas, el Círculo Social significa diversión, distracción, un lugar para estar con los amigos, así lo manifestó $68 \%$ de los entrevistados, mientras que $20.5 \%$ lo asocia a alcoholismo y problemas, y para $11.5 \%$ no significa nada, o simplemente no respondieron al respecto, mientras que para los representantes de la comunidad es el lugar con el que pueden contar para realizar reuniones.

\section{Opinión}

En el cuestionario, en cuanto al punto referente a definir qué es la recreación de la comunidad, solo $24.4 \%$ 
considera que es el conjunto de actividades culturales y deportivas programadas para todos los grupos de edades, en las que se pueden desarrollar y divertir sanamente; $64.1 \%$ de los encuestados valora la recreación como bailar, ir a fiestas, tomar bebidas alcohólicas, disfrutar un buen ambiente, "despejar la mente", divertirse. Para la población de la tercera edad la recreación consiste en estar con la familia, hacer actividades en la casa, ver televisión o pasear, lo que representó el $10.2 \%$. Hubo una persona que opinó que la recreación es mala e insignificante, opinión sin relevancia para esta investigación ya que frente a las demás se puede comprobar que la recreación sí es relevante. De las personas encuestadas, $83.3 \%$ sostuvo que la recreación es muy importante porque se realizan diferentes actividades para que las personas se diviertan y motiven, mejora la calidad de vida $\mathrm{y}$ hace que estas sean felices aunque sea por un rato y se olviden de sus problemas.

También se opina que el Círculo Social Obrero "Pedro Soto Alba" es un lugar donde se hacen actividades para que se diviertan las personas de menos recursos, se puede pasar un rato agradable con los amigos y celebrar cumpleaños; además se insiste en que es el único lugar de la comunidad donde puede ir la juventud a divertirse, aunque las personas que más participan y asisten a él tienen edades comprendidas entre los 29 y los 50 años, generalmente en las tardes y los fines de semana, para "descansar después del trabajo", quienes afirmaron esto representan $74.4 \%$ de la muestra. El $21.7 \%$ de los entrevistados valora el Círculo Social como el núcleo donde las personas ingieren bebidas alcohólicas en grandes cantidades, así que no lo consideran un buen sitio para divertirse, porque pueden estar expuestos a algún tipo de problema debido a la poca seguridad que ofrece.

Comparando estas opiniones, salta a la vista por qué muchas personas son asiduas al lugar, y aunque este no hace grandes ofertas recreativas, estas satisfacen los requerimientos de muchos habitantes para quienes la recreación consiste solo en pasar un rato agradable con los amigos, tomarse una cerveza o escuchar música.

También se ilustran las cualidades con las que las personas designaron el Círculo Social, al opinar sobre él aportando valoraciones, juicios y reflexiones; para los habitantes del 
sector es de suma importancia que sus opiniones sean escuchadas con el fin de que se mantenga este espacio, no como ahora, sino cada día mejor, y pueda llegar a convertirse en un núcleo fuerte y representativo de la recreación comunitaria, que la fortalezca y a la vez mejore su imagen en el imaginario colectivo.

Como se puede apreciar, la mayoría (44.8 \%) tiene buena opinión del lugar, a la que se suma $11.5 \%$ de quienes elevan esa categoría a muy buena; $21.7 \%$ juzga que es regular, $16.6 \%$ que es malo y $5.1 \%$ muy malo (tanto por las condiciones físicas que exhibe, como por las personas que lo frecuentan). Cuando se indagó qué era lo mejor del club, los entrevistados respondieron de múltiples maneras: por ejemplo, $15.3 \%$ opina que es el lugar con el que se cuenta; para $30.7 \%$ lo mejor es el conjunto de servicios que se prestan, las actividades, la música, la cerveza, la atención y el buen trato de los trabajadores; $19.2 \%$ ve en la cercanía su mejor cualidad, así que pueden divertirse en las proximidades de sus viviendas sin tener que transportarse; $8.9 \%$ afirma que no hay nada bueno, todo es malo; por el contrario, $11.5 \%$ sostiene que todo es bueno; otro 11.5 \% no opinó, dejó la pregunta sin contestar.

$\mathrm{Al}$ inquirir por lo peor del Círculo Social, las opiniones también fueron variadas: $29.4 \%$ cree que las malas condiciones del lugar, lo feo que se encuentra, la ausencia de ofertas gastronómicas, la música, la falta de bebidas en algunas ocasiones y la entrada al lugar, porque plantean que deben pagarla varias veces.

Un $17.9 \%$ opina que lo peor son las personas que frecuentan el lugar y consumen bebidas alcohólicas en exceso, lo que genera problemas en ocasiones; $5.1 \%$, que no se usa debidamente, puesto que se puede aprovechar más con la realización de actividades culturales y un horario más prolongado.

Uno de los encuestados expresó: “¿Lo peor? Que no está disponible todo el día"; $20.5 \%$ asevera que allí todo es bueno, mientras que $8.9 \%$ plantea lo contrario; $17.9 \%$ no supo contestar la pregunta o esta se dejó en blanco.

Cuando se pidió que escribieran las palabras que se les viniera a la mente cuando se hablaba del Círculo Social Obrero "Pedro Soto Alba", estas en su mayoría fueron: actividad, música, bebida, cumpleaños, amigos, tranquilidad, fiesta, 
recreación, diversión; con esto se puede comprobar cómo el club cumple en parte con la recreación que demanda su público. Sin embargo, cuando se preguntó qué necesitaba el Círculo Social, $60.2 \%$ considera que recursos materiales para arreglarlo, invertir en él para mejorar sus condiciones físicas; 10.2 $\%$, que realizar actividades culturales y deportivas para niños, jóvenes y personas de la tercera edad; $11.5 \%$ quisiera ofertas gastronómicas; 3.8 $\%$ aduce que no necesita nada; 2.5 $\%$, que bajar el precio de la entrada; $7.6 \%$ no contestó.

$\mathrm{Al}$ referirse a lo que necesita en recreación la comunidad para motivarse a vivir allí la opinión predominante (91\%) fue de tipo material, como parques recreativos, un campo de fútbol, piscina, pista de carreras, planes de calle semanales, talleres de creación, una discoteca, fiestas populares, grupos de teatro callejero y actividades con música los fines de semana.

La valoración de los habitantes acerca del Círculo Social es esencialmente la de ser un lugar importante para la comunidad en el que se pueden desarrollar disímiles actividades para que las personas de todas las edades se diviertan; es el único lugar creado para la recreación y es asequible a las posibilidades económicas de los residentes porque el salario promedio del lugar es bajo y muchas personas se encuentran laborando en empleos no formales; además, se puede explotar con actividades culturales o deportivas y ofertas, incluyendo la de gastronomía, tan necesaria y solicitada por los pobladores.

\section{Actitud}

Respecto al conocimiento acerca de la recreación en general de la circunscripción, $62 \%$ no sabe cuáles son las actividades que se realizan para proporcionar recreación a sus habitantes. Los que respondieron que sí las conocían representaron $37.1 \%$ y de estos todos dijeron que se trataba de actividades en fechas especiales como las del 28 de septiembre, cuando se hacen intercambios de regalos, se llevan platos de dulces y se celebran actividades culturales y deportivas por parte de Cultura y el Instituto Nacional de Deportes, Educación Física y Recreación (Inder).

En entrevista con el delegado, este plantea que sí se realizan las actividades por parte de Cultura y el Inder, pero no se puede llegar a toda 
la circunscripción porque es muy amplia y necesitan de más recursos; no basta con solo el interés de los promotores, sino que se requieren equipos de audio, materiales e instrumentos deportivos.

En la parte cognitiva se puede apreciar que todos los encuestados conocen el Círculo, su objeto social, su horario, las actividades que lleva a cabo y todo lo relacionado con él; aunque sea solo una vez en la vida, lo han visitado y disfrutado de sus servicios; debido a este conocimiento más o menos profundo en cada uno de los casos es que pueden emitir juicios y valoraciones, porque de alguna manera ya se ha conformado una imagen mental del lugar de acuerdo con las representaciones adquiridas mediante los sentidos.

La recreación es para la comunidad citada algo muy apreciado, en la parte afectiva la ven como algo importante pues ayuda a que los vecinos se relacionen y surjan lazos de compañerismo y amistad entre ellos, se comuniquen mejor, lo cual contribuye a elevar el nivel cultural de las personas y fortalece el sentimiento de comunidad.

La circunscripción objeto de estudio ha sido formada casi en su totalidad por inmigración, asentamientos que nacen de un día para otro con grupos de personas que no se conocen y que a partir de ese momento comienzan a establecer un proyecto de vida en común para ir conformando su propia historia, de manera que la recreación constituye parte de esa historia porque los une, no por planes administrativos ni geográficos, sino por la necesidad que tienen de diversión y esparcimiento.

Afectivamente se puede constatar que los habitantes de la comunidad sienten aprecio y sentido de pertenencia hacia el club, lo consideran un lugar al que pueden y quieren ir; les gustaría sí que lo arreglaran, sienten nostalgia por lo que fue en años anteriores; uno de los encuestados manifestó: "quisiera que fuera como hace diez años".

Se puede valorar cómo en lo conductual cambia la situación, porque muchos, aunque tienen buenos recuerdos del club, ya no asisten a él; $30.7 \%$ manifiesta que no lo visitan por las condiciones actuales en las que se halla; $59 \%$ asiste a las actividades que se realizan allí para divertirse con los amigos y establecer nuevas relaciones sociales, tomarse una cerveza u oír música, se sienten motivados por asistir a él. Uno de 
los encuestados dijo que "le permitía ser 'un alcohólico', lo cual no es una conducta a imitar", y $8.9 \%$ no opinó.

La conducta de las personas que participan en las actividades del Círculo Social se observó adecuada, dentro de un ambiente agradable en el que los asistentes, aunque ingieran bebidas alcohólicas, conversan sin bullicio, oyen música y, en algunas ocasiones, bailan.

\section{Mediaciones sociales}

Las mediaciones individuales presentes en la imagen pública son la edad, el sexo, la ocupación laboral y el nivel de escolaridad de los encuestados y entrevistados.

Se pudo constatar que la población infantil solo asiste al club a cumpleaños de otros niños, de ahí que tienen poco conocimiento de las actividades que allí se realizan y generalmente lo ven como un lugar bonito porque en estos casos lo encuentran decorado con globos, cadenetas y manteles.

Las personas entre los 15 y 30 años no asisten regularmente; ellos, para su entretenimiento, desean otras actividades; cuando a esta población se le preguntó qué necesitaba el club, contestó que juegos de luces, música actual, espectáculos de variedades o una discoteca. Este grupo de edades también asiste cuando se hacen fiestas quinceañeras y lo ven adornado, bonito. Las personas con edades entre 30 y 60 años sí participan regularmente y lo que más les gusta es la música y el poder conversar sentados tranquilamente con los amigos. Para las personas mayores de 60 años lo fundamental es que pueden ir a comprar la cerveza para tomarla en casa, poco participan activamente en el club.

Quienes más participan en las actividades son los hombres, solo van algunas mujeres, principalmente los fines de semana. Uno de los encuestados, al preguntársele qué necesitaba el club, respondió: "Más mujeres".

El nivel cultural de la población de esta comunidad es bajo: $10.2 \%$ apenas ha cursado el nivel primario; $47.4 \%$ tiene aprobado el nivel secundario; $24.4 \%$ acredita carrera técnica media o grado 12 y solo $17.9 \%$ de los encuestados posee título universitario.

Entre las mediaciones textuales está la tecnológica, a partir del lenguaje y los atributos técnicos del medio; en el caso de este estudio no hay tecnología en el lugar donde se desarrollan los procesos, sino que 
estos se realizan por medio de la comunicación interpersonal.

Entre la administración del Círculo Social y los representantes de la comunidad existen buenas relaciones y socializan en las reuniones del consejo popular, en las del grupo de trabajo comunitario y en las de coordinación, según manifestaron todos los entrevistados.

Las mediaciones contextuales incluyen la relación entre mediaciones microsociales y macrosociales. En las mediaciones microsociales se contemplan las relaciones del sujeto con su familia, sus vecinos y comunidad. En este caso de estudio, es una comunidad altamente marginal debido al bajo nivel económico de sus habitantes y las malas condiciones de vida, que provocan problemas entre ellos, lo cual también ocasiona que las personas se dirijan al club a ingerir bebidas alcohólicas como una manera de olvidar sus problemas cotidianos.

En las mediaciones macrosociales se tiene en cuenta la religión, en la que son fundamentales los cultos protestantes, aunque no existe Iglesia alguna constituida; esta es una de las causas por la que algunas personas no asisten al club, ya que este tipo de religión prohíbe las bebidas alcohólicas. Las organizaciones políticas y sociales cumplen con su función de promover la recreación sana, sin embargo, muchas veces no llega a ser percibida por los habitantes porque no representa el tipo de actividades que prefieren o se dificultan los recursos materiales para realizarlas de la mejor calidad.

En la mediación situacional se debe señalar que las encuestas aplicadas se llevaron a cabo principalmente en las calles y portales de las viviendas, y catorce de ellas se aplicaron a personas que disfrutaban de los servicios del club, pero, que viven en la circunscripción.

Cabe indicar que una mediación importante, aunque no figura entre las catalogadas, son las mediaciones comunicativas; el sistema de comunicación puede utilizarse como un interventor para que controle el funcionamiento de alguna institución o fenómeno social como la recreación. Mediante la comunicación se puede intervenir en las necesidades, los valores y los modelos del mundo que caracterizan a los diferentes grupos humanos, utilizándose como mediaciones comunicativas.

En el caso de esta investigación, debido al bajo índice de escolaridad 
muchos de los encuestados no supieron escribir en el papel todo lo que deseaban expresar, por lo que también se tomaron en consideración sus expresiones verbales mientras llenaban los cuestionarios.

Una de las problemáticas planteadas y que toca el tema de la comunicación fue la escasa divulgación de las actividades, debido a lo cual muchas personas no participan porque no saben que se va a realizar una actividad, además no se les motiva con pancartas y material informativo.

De todas las mediaciones estudiadas, sin dudas la más relevante se encuentra en el plano individual, al constatar cómo las edades influyen de manera determinante en la participación activa en las dinámicas que propone el Círculo Social.

\section{Resumen}

$\mathrm{Al}$ analizar el conjunto de los cuestionarios y valorar las respuestas sobre cada una de las dimensiones contempladas en este estudio, e incluir además los conocimientos aportados a esta tesis por la observación en las áreas del Círculo Social Obrero "Pedro Soto Alba" y en general en la demarcación de la circunscripción 144 , se pudo determinar cómo se comporta la participación de sus habitantes en este club.

Se resalta que los mayores niveles de participación se ubican en las edades comprendidas entre los 30 y 60 años, esto debido a que dichas personas se sienten más cansadas de la vida diaria, de ahí que desean pasar un rato agradable, olvidando los problemas cotidianos de la casa, el trabajo y la familia. De ahí, expresiones como: "Nos ayuda a despejar la mente de manera tal que nos permite dejar atrás por un rato los problemas familiares y personales", o "Hay muchos ciudadanos que después de tener una larga semana de trabajo pueden ir a recrearse los sábados y domingos".

Los niños solo participan a veces, cuando asisten a cumpleaños de otros niños; debido al expendio de bebidas alcohólicas no se les permite la entrada ni siquiera en horarios diurnos y hasta el momento no se ha creado ningún espacio regular para su sano esparcimiento; únicamente en las vacaciones se realizan actividades para la población infantil, entre las que se encuentra el Plan de Verano, con payasos y juegos participativos.

Por su parte, los jóvenes tampoco participan con mucha frecuencia, 
pues para su recreación prefieren lugares más de moda, donde puedan compartir con otras personas de su edad y disfrutar de sus mismas preferencias, como la música del momento. Ellos también demandan más recursos materiales, como mejores equipos de audio y juegos de luces.

Las personas de la tercera edad deben su escasa participación a que en materia de recreación sus gustos son bien distintos, plantean que su mejor diversión reside en estar con la familia, realizar fiestas en el hogar y ver televisión, el mayor uso que le dan al club es que van allí a comprar la cerveza para acompañar alguna actividad festiva en casa.

Se puede decir, a partir de todo lo anterior, que la imagen del Círculo Social Obrero "Pedro Soto Alba" se traduce en que este es un espacio de recreación que brinda diversión a los habitantes de la circunscripción 144 del Consejo Popular La Belén mediante la venta de cerveza y la música grabada. Sin embargo, no cuenta con generalizada participación de la comunidad y necesita de otros tipos de actividades de tipo cultural y ofertas gastronómicas para poder complacer a todos los grupos etáreos y lograr una recreación sana en esta comunidad que tanto la necesita.

También influyen en la conformación de la imagen de dicho club la dinámica de vida de los habitantes de la circunscripción, sus gustos y preferencias, para así poder elaborar propuestas de recreación ajustadas a las necesidades de cada grupo.

Las instituciones y los representantes del poder popular, al hacer una oferta de participación, están en situación de dependencia frente al público, así que sus ofertas de participación deben ser atractivas para la población, de acuerdo con sus intereses, si en verdad esperan recibir una respuesta favorable.

\section{Discusión}

La imagen de la recreación que tienen los habitantes de la comunidad perteneciente a la circunscripción 144 del Consejo Popular La Belén no se ha formado aún del todo, no existe un programa organizado que contribuya a su desarrollo, solo se realizan acciones aisladas con pocos recursos y en ocasiones no se les da la divulgación requerida, de modo que las personas no se enteran y las actividades apenas son disfrutadas por un grupo minoritario. 
La imagen pública que se tiene del Círculo Social Obrero "Pedro Soto Alba”, institución responsable de proveer a los pobladores de la circunscripción 144 del Consejo Popular La Belén la recreación que necesitan, es la de ser un lugar destinado a proporcionar diversión por medio de la venta de bebidas alcohólicas y la música, además de un lugar tranquilo donde se puede pasar un rato agradable con los amigos.

\section{Referencias}

Casales, J. C. (2009). Conocimientos básicos de psicología social. Selección de lecturas. La Habana: Félix Varela.

Manzano, M. (2006). Introducción a la percepción. La Habana: Félix Varela.

Portal, R. y Recio, M. (2004). Lecturas sobre comunicación social. La Habana: Félix Varela.

Trelles, I.; Meriño J. y Espinoza A. (2005). Comunicación, imagen e identidad corporativas. Selección de lecturas. La Habana: Félix Varela.

Delvalle, L. (2007). Diagnóstico de la participación juvenil en el Taller de transformación integral del barrio Príncipe (trabajo de grado). La Habana: Universidad de La
Habana-Licenciatura en Comunicación Social.

Díaz, M. (2009). Mil palabras, una imagen, una aproximación al diagnóstico de la imagen pública del Taller de transformación integral de barrio de la comunidad de Micro X del Consejo Popular Alamar del Este (trabajo de grado). La Habana: Universidad de La Habana-Licenciatura en Comunicación Social.

Milán, A. y Noval, L. de la (2007). Aproximación al diagnóstico de la identidad e imagen del Programa de Trabajadores Sociales de Arroyo Naranjo (trabajo de grado). La Habana: Universidad de La Habana-Licenciatura en Comunicación Social.

Montes de Oca, J. (2010). Estrategia de comunicación para fortalecer la identidad corporativa del Ballet de Camagüey (trabajo de grado). Camagüey, Cuba: Universidad de Camagüey-Licenciatura en Comunicación Social.

Suárez, E. (2010). Estrategia de comunicación para fortalecer la autoimagen corporativa de la compañía Danza Contemporánea de Cuba (trabajo de grado). Camagüey, Cuba: Universidad de Camagüey-Licenciatura en Comunicación Social. 


\section{Apéndice A}

Tabla A1. Distribución del muestreo intencional por cuotas. Distribución de las 78 personas encuestadas por rango de edad y sexo

\begin{tabular}{|c|c|c|c|}
\hline Edad & Masculino & Femenino & Total \\
\hline $9-14$ & 3 & 2 & 5 \\
\hline $15-19$ & 3 & 2 & 5 \\
\hline $20-24$ & 3 & 3 & 6 \\
\hline $25-29$ & 4 & 4 & 8 \\
\hline $30-39$ & 8 & 10 & 18 \\
\hline $40-49$ & 6 & 6 & 12 \\
\hline $50-59$ & 5 & 5 & 10 \\
\hline $60 y+$ & 6 & 8 & 14 \\
\hline Total & $\mathbf{3 8}$ & $\mathbf{4 0}$ & $\mathbf{7 8}$ \\
\hline
\end{tabular}

